PROCEEDINGS OF THE AMERICAN MATHEMATICAL SOCIETY

Volume 124, Number 1, January 1996

\title{
A RIGIDITY THEOREM FOR THE CLIFFORD TORI IN $S^{3}$
}

\author{
KAZUYUKI ENOMOTO, YOSHIHISA KITAGAWA, AND JOEL L. WEINER
}

(Communicated by Christopher Croke)

\begin{abstract}
Let $S^{3}$ be the unit hypersphere in the 4-dimensional Euclidean space $\mathbb{R}^{4}$ defined by $\sum_{i=1}^{4} x_{i}^{2}=1$. For each $\theta$ with $0<\theta<\pi / 2$, we denote by $M_{\theta}$ the Clifford torus in $S^{3}$ given by the equations $x_{1}^{2}+x_{2}^{2}=\cos ^{2} \theta$ and $x_{3}^{2}+x_{4}^{2}=\sin ^{2} \theta$. The Clifford torus $M_{\theta}$ is a flat Riemannian manifold equipped with the metric induced by the inclusion map $i_{\theta}: M_{\theta} \rightarrow S^{3}$. In this note we prove the following rigidity theorem: If $f: M_{\theta} \rightarrow S^{3}$ is an isometric embedding, then there exists an isometry $A$ of $S^{3}$ such that $f=A \circ i_{\theta}$. We also show no flat torus with the intrinsic diameter $\leq \pi$ is embeddable in $S^{3}$ except for a Clifford torus.
\end{abstract}

\section{INTRODUCTION}

Let $S^{3}$ be the unit hypersphere in the 4-dimensional Euclidean space $\mathbb{R}^{4}$ defined by $\sum_{i=1}^{4} x_{i}^{2}=1$. For each $\theta$ with $0<\theta<\pi / 2$, we denote by $M_{\theta}$ the Clifford torus in $S^{3}$ given by

$$
x_{1}^{2}+x_{2}^{2}=\cos ^{2} \theta, \quad x_{3}^{2}+x_{4}^{2}=\sin ^{2} \theta .
$$

The Clifford torus $M_{\theta}$ is a flat Riemannian manifold equipped with the metric induced by the inclusion map $i_{\theta}: M_{\theta} \rightarrow S^{3}$. The authors are interested in the following question: For every isometric immersion $f: M_{\theta} \rightarrow S^{3}$, does there exist an isometry $A$ of $S^{3}$ such that $f=A \circ i_{\theta}$ ? Concerning this question, it is known that if $f_{t}: M_{\theta} \rightarrow S^{3},-\infty<t<\infty$, is a smooth 1-parameter family of isometric immersions with $f_{0}=i_{\theta}$, then for each $t$ there exists an isometry $A_{t}$ of $S^{3}$ such that $f_{t}=A_{t} \circ i_{\theta}$. However, the question above seems not to have been settled yet. In this note we give an affirmative answer to the question under the assumption that the immersion $f$ is an embedding. In other words, we prove the following rigidity theorem.

Theorem 1. If $f: M_{\theta} \rightarrow S^{3}$ is an isometric embedding, then there exists an isometry $A$ of $S^{3}$ such that $f=A \circ i_{\theta}$.

For each isometric immersion $f: M_{\theta} \rightarrow S^{3}$, we denote by $\operatorname{Diam}(f)$ the diameter of the image $f\left(M_{\theta}\right)$ in $S^{3}$. Note that $\operatorname{Diam}\left(i_{\theta}\right)=\pi$. The following theorem, which will be proved in $\S 2$, is a key ingredient in the proof of Theorem 1 .

Received by the editors December 16, 1993 and, in revised form, July 7, 1994.

1991 Mathematics Subject Classification. Primary 53C40; Secondary 53C45.

Key words and phrases. Clifford torus, flat torus. 
Theorem 2. If $f: M_{\theta} \rightarrow S^{3}$ is an isometric immersion with $\operatorname{Diam}(f)=\pi$, then there exists an isometry $A$ of $S^{3}$ such that $f=A \circ i_{\theta}$.

We now give the proof of Theorem 1. It follows from [2] that if $f$ is an isometric embedding of a flat torus $M$ into $S^{3}$, then the image $f(M)$ is invariant under the antipodal map of $S^{3}$. In particular, for each isometric embedding $f: M_{\theta} \rightarrow S^{3}$, we have $\operatorname{Diam}(f)=\pi$. Therefore the assertion of Theorem 1 follows from Theorem 2 .

In $\S 3$, we obtain Theorem 3, which generalizes Theorem 2 for an isometric immersion of a flat torus with intrinsic diameter less than or equal to $\pi$. An immediate consequence of Theorem 3 is that the only flat tori with intrinsic diameter $\leq \pi$ which can be embedded in $S^{3}$ are Clifford tori.

\section{Proof of Theorem 2}

We define a Riemannian covering map $T: \mathbb{R}^{2} \rightarrow M_{\theta}$ of the 2-dimensional Euclidean space $\mathbb{R}^{2}$ into the Clifford torus $M_{\theta}$ by setting

$$
T\left(u_{1}, u_{2}\right)=\left(R_{1} \cos \left(\frac{u_{1}}{R_{1}}\right), R_{1} \sin \left(\frac{u_{1}}{R_{1}}\right), R_{2} \cos \left(\frac{u_{2}}{R_{2}}\right), R_{2} \sin \left(\frac{u_{2}}{R_{2}}\right)\right),
$$

where $R_{1}=\cos \theta$ and $R_{2}=\sin \theta$. Note that $T\left(u_{1}, u_{2}\right)=T\left(u_{1}+l_{1}, u_{2}+l_{2}\right)$ if and only if $l_{i} / 2 \pi R_{i}$ is an integer for each $i$. Let $V_{1}$ and $V_{2}$ be the vector fields on $M_{\theta}$ given by

$$
\left\{\begin{array}{l}
V_{1}\left(T\left(u_{1}, u_{2}\right)\right)=\left.\frac{d}{d t}\right|_{t=0} T\left(u_{1}+R_{1} t, u_{2}+R_{2} t\right), \\
V_{2}\left(T\left(u_{1}, u_{2}\right)\right)=\left.\frac{d}{d t}\right|_{t=0} T\left(u_{1}+R_{1} t, u_{2}-R_{2} t\right)
\end{array}\right.
$$

Then we have

$$
g\left(V_{1}, V_{1}\right)=g\left(V_{2}, V_{2}\right)=1, \quad g\left(V_{1}, V_{2}\right)=\cos 2 \theta,
$$

where $g$ denotes the Riemannian metric on $M_{\theta}$. For $i=1$, we denote by $\left\{\varphi_{i}^{t}\right\}$ the 1-parameter group of transformations of $M_{\theta}$ generated by the vector field $V_{i}$.

Lemma 1. Let $f: M_{\theta} \rightarrow S^{3}$ be an isometric immersion, and let $p$ be a point in $M_{\theta}$. If there exists a point $q \in M_{\theta}$ such that $f(p)=-f(q)$, then the curve $\gamma_{i}(t)=f\left(\varphi_{i}^{t}(p)\right)$ is a unit speed geodesic in $S^{3}$.

Proof. Take a point $\left(a_{1}, a_{2}\right) \in \mathbb{R}^{2}$ such that $T\left(a_{1}, a_{2}\right)=p$. By (1) we obtain

$$
\left\{\begin{array}{l}
\varphi_{1}^{t}(p)=T\left(a_{1}+R_{1} t, a_{2}+R_{2} t\right) \\
\varphi_{2}^{t}(p)=T\left(a_{1}+R_{1} t, a_{2}-R_{2} t\right)
\end{array}\right.
$$

Let $d(p, q)$ denote the intrinsic distance between $p$ and $q$ in $M_{\theta}$. Then it follows from $f(p)=-f(q)$ that $d(p, q) \geq \pi$. Since the intrinsic diameter of $M_{\theta}$ is equal to $\pi$, we obtain $d(p, q)=\pi$. Hence

$$
q=T\left(a_{1}+R_{1} \pi, a_{2}+R_{2} \pi\right)=T\left(a_{1}+R_{1} \pi, a_{2}-R_{2} \pi\right) .
$$

It follows from (3) and (4) that $\gamma_{i}(t)$ is a unit speed curve in $S^{3}$ such that $\gamma_{i}(0)=$ $\gamma_{i}(2 \pi)=f(p)$ and $\gamma_{i}(\pi)=f(q)=-f(p)$. This shows that $\gamma_{i} \mid[0,2 \pi]$ is a geodesic in $S^{3}$. Since $\gamma_{i}(t+2 \pi)=\gamma_{i}(t)$, the curve $\gamma_{i}(t)$ is a unit speed geodesic in $S^{3}$.

Lemma 2. Let $f: M_{\theta} \rightarrow S^{3}$ be an isometric immersion with $\operatorname{Diam}(f)=\pi$, and let $h$ denote the second fundamental form of the immersion $f$. Then $h\left(V_{1}, V_{1}\right)=$ $h\left(V_{2}, V_{2}\right)=0$ and $\left|h\left(V_{1}, V_{2}\right)\right|=\sin 2 \theta$. 
Proof. We set $h_{i j}=h\left(V_{i}, V_{j}\right)$. By (2) and the equation of Gauss, we obtain

$$
\left\langle h_{12}, h_{12}\right\rangle-\left\langle h_{11}, h_{22}\right\rangle=\sin ^{2} 2 \theta,
$$

where $\langle$,$\rangle denotes the Riemannian metric on S^{3}$. We now define $M_{\theta}^{*}$ to be the set of all $p \in M_{\theta}$ such that $f(p)=-f(q)$ for some $q \in M_{\theta}$. Using Lemma 1, we see that $h_{11}=h_{22}=0$ on $M_{\theta}^{*}$. So it is sufficient to show that $M_{\theta}^{*}=M_{\theta}$. Since $\operatorname{Diam}(f)=\pi$, there exists a point $p \in M_{\theta}^{*}$. Let $c(s)$ be the curve in $M_{\theta}$ given by $c(s)=\varphi_{1}^{s}(p)$. Then it follows from Lemma 1 that the curve $f(c(s))$ is a unit speed geodesic in $S^{3}$, and so $f(c(s))=-f(c(s+\pi))$. Hence $c(s) \in M_{\theta}^{*}$ for all $s$. For each $s \in \mathbb{R}$, let $c_{s}(t)$ be the curve in $M_{\theta}$ given by $c_{s}(t)=\varphi_{2}^{t}(c(s))$. By the same way as above we see that $c_{s}(t) \in M_{\theta}^{*}$. Hence $\varphi_{2}^{t}\left(\varphi_{1}^{s}(p)\right) \in M_{\theta}^{*}$ for all $(s, t) \in \mathbb{R}^{2}$. This implies $M_{\theta}^{*}=M_{\theta}$.

We now give the proof of Theorem 2. Let $f: M_{\theta} \rightarrow S^{3}$ be an isometric immersion with $\operatorname{Diam}(f)=\pi$. We set $f_{1}=i_{\theta}$ and $f_{2}=f$. For $k=1,2$, let $h_{k}$ be the second fundamental form of the immersion $f_{k}$, and let $\xi_{k}=h_{k}\left(V_{1}, V_{2}\right) / \sin 2 \theta$. Then it follows from Lemma 2 that $\xi_{k}$ defines a unit normal vector field along $f_{k}$, and

$$
\left\langle h_{1}\left(V_{i}, V_{j}\right), \xi_{1}\right\rangle=\left\langle h_{2}\left(V_{i}, V_{j}\right), \xi_{2}\right\rangle .
$$

Hence the fundamental theorem of the theory of surfaces implies that there exists an isometry $A$ of $S^{3}$ such that $f_{2}=A \circ f_{1}$. This completes the proof of Theorem 2 .

\section{A generalization of Theorem 2}

In this section we generalize Theorem 2 as follows.

Theorem 3. Let $M$ be a flat torus with intrinsic diameter less than or equal to $\pi$. If $f: M \rightarrow S^{3}$ is an isometric immersion with $\operatorname{Diam}(f)=\pi$, then there exist an isometry $\varphi: M_{\theta} \rightarrow M$ for some $\theta \in(0, \pi / 2)$ and an isometry $A$ of $S^{3}$ such that $f \circ \varphi=A \circ i_{\theta}$.

Proof. Since $\operatorname{Diam}(f)=\pi$, there exist points $p, q$ in $M$ such that $f(p)=-f(q)$. Let $d(p, q)$ denote the intrinsic distance between $p$ and $q$ in $M$. It follows from $f(p)=-f(q)$ that $d(p, q) \geq \pi$. But our assumption on the intrinsic diameter of $M$ implies that $d(p, q)=\pi$. In addition, any geodesic of $M$ of length $\pi$ which connects $p$ and $q$ is mapped by $f$ to a geodesic $S^{3}$ which connects $f(p)$ to $f(q)$.

If $M$ is not isometric to $M_{\theta}$, we claim that $p$ and $q$ are connected by three geodesics in $M$ of length $\pi$ whose tangent vectors at $p$ lie in three distinct linear subspaces. To prove the claim consider the Riemannian covering map $k: \mathbb{R}^{2} \rightarrow M$. We suppose that 0 , the origin, lies in $\Gamma=k^{-1}(q)$; of course, $M$ is isometric to $\mathbb{R}^{2} / \Gamma$. Let $q_{1}$ and $q_{2}$ be the elements of smallest norm in $\Gamma \backslash\{0\}$ and $\Gamma \backslash\left\{n q_{1}: n \in \mathbb{Z}\right\}$, respectively. For notational reasons, let $0=q_{0}$. Denote the triangle with vertices $q_{i}$, for $i=0,1,2$, by $\Delta$. One may show that the circumcenter of $\Delta$, denote $p_{0}$, lies in $k^{-1}(p)$ and $d\left(p_{0}, q_{i}\right)=\pi$, for $i=0,1,2$. Thus there are at least three geodesics in $M$ of length $\pi$ connecting $p$ to $q$. Assume that the tangent vectors to these geodesic arcs at $p$ must lie in a pair of linear subspaces of the tangent space to $M$ at $p$. Necessarily, two of the segments $\overline{p_{0} q_{i}}$ lie on the same line. Say, for example, that the segment $\overline{q_{1} q_{2}}=\overline{q_{1} p_{0}} \cup \overline{p_{0} q_{2}}$. Since the circumcenter of $\Delta$ is on the side $\overline{q_{1} q_{2}}$ of $\Delta$, one sees that $\Delta$ is a right triangle with right angle at $q_{0}$. It follows that $M$ is isometric to a Clifford torus. This contradiction proves the claim.

If $M$ is not isometric to $M_{\theta}$, then the images under $f$ of the geodesic segments mentioned in the previous paragraph are geodesics of $S^{3}$. This implies that the 
immersion $f$ is totally geodesic at $p$, which is impossible. Hence $M$ must be intrinsically isometric to $M_{\theta}$. Now Theorem 3 follows from Theorem 2.

Theorem 4. It is impossible to embed a flat torus with intrinsic diameter $\leq \pi$ in $S^{3}$ unless the flat torus is a Clifford torus.

Proof. Again, from [2], the image of any embedding must have antipodal symmetry. Thus the assertion of this theorem follows from Theorem 3.

\section{REFERENCES}

1. Y. Kitagawa, Rigidity of the Clifford tori in $S^{3}$, Math. Z. 198 (1988), 591-599. MR 89g:53081 2. _ Embedded flat tori in the unit 3-sphere J. Math. Soc. Japan 47 (1995), 275-296.

Faculty of Industrial Science and Technology, Science University of Tokyo, OsHAMANBE, HoKKAIDO, 049-35 JAPAN

E-mail address: enomoto@it.osha.sut.ac.jp

Department of Mathematics, Utsunomiya University, Mine-machi, Utsunomiya, 321 JAPAN

Department of Mathematics, University of Hawail at Manoa, 2565 The Mall, HonOLULU, HaWAiI, 96822 U.S.A.

E-mail address: joel@math.hawaii.edu 